\title{
Prognostic Implications of 'Paced' and 'Native' QRS Durations Following Cardiac Resynchronization Therapy
}

\author{
Oguz Karaca, MD
}

Cardiology Department, Medipol University Faculty of Medicine, Istanbul, Turkey

DOI: http://doi.org/10.17925/EJAE.2016.02.01.30

\begin{abstract}
Current evidence strongly suggests that the extent of electrical dyssynchrony within the left ventricle is determined by the delayed intraventricular conduction time reflected by a prolonged QRS duration (QRSd) on the surface (ECG). However, in cardiac resynchronization therapy (CRT) follow-up algorithms, the QRSd on the post-operative ECG has been relatively less frequently addressed, although the baseline QRSd is accepted as an essential 'pre-operative' marker for patient selection and prediction of response to therapy. In this review, we discuss the clinical impact of post-implantation electrocardiographic parameters, such as the 'paced' QRSd and 'native' QRSd (assessed when the device is temporarily switched off) on the efficacy of therapy and on prediction of future outcomes after CRT.
\end{abstract}

\section{Keywords}

Cardiac resynchronization therapy, QRS duration, prognosis

\begin{abstract}
Disclosure: Oguz Karaca, MD, has nothing to disclose in relation to this article. No funding was received in the publication of this article. open Access: This article is published under the Creative Commons Attribution Noncommercial License, which permits any noncommercial use, distribution, adaptation, and reproduction provided the original author(s) and source are given appropriate credit. Received: 29 February 2016 Accepted: 6 April 2016 Citation: European Journal of Arrhythmia \& Electrophysiology, 2016;2(1):30-2 Correspondence: Oguz Karaca, MD, TEM Otoyolu Göztepe çıkıII, No:1, Bağclar İstanbul, Turkey. E: oguzkaraca@hotmail.com
\end{abstract}

\section{Current use of QRS duration in cardiac resynchronization therapy}

Cardiac resynchronization therapy (CRT) is an established therapeutic option in patients with symptomatic systolic heart failure concomitant with electrical dyssynchrony. ${ }^{1-3}$ Patients with a wide QRS ( $\left.\geq 120 \mathrm{~ms}\right)$, especially those with a left bundle branch block (LBBB) morphology, derive the most benefit from biventricular pacing in terms of functional and echocardiographic response. ${ }^{3}$ CRT is clearly an electrical therapy that aims to restore the synchronicity (timing of the onset of contraction) of the opposing myocardial segments within the left ventricle. Following device implantation, the magnitude of response to therapy has been conventionally assessed with improvements in functional capacity or in left ventricular (LV) mechanics (increase in LV ejection fraction or decrease in LV end-systolic volume). 4,5 However, in the current CRT follow-up algorithms, the QRSd on the post-operative electrocardiogram (ECG; assessed while both pacing on and pacing off) has been relatively less frequently addressed for the purpose of determining adequate therapy in relation to clinical outcomes.

\section{Paced QRS duration in patients with heart failure}

Given the strong evidence derived from the right ventricular (RV) pacing studies in heart failure patients, a prolonged QRSd on the paced ECG is associated with a poor outcome, including deterioration of LV function and the development of decompensation in heart failure (even in patients with previously normal systolic function), ${ }^{6,7}$ The investigators of the PREDICT-HF trial concluded that RV pacing-induced QRS prolongation reflected poor outcome in terms of worsening LV systolic function and increased rate of heart failure events. ${ }^{7}$ The clinical association of RV pacing and worse outcomes in heart failure population is basically explained by the stimulation of 'iatrogenic' electrical dyssynchrony by means of deterioration of the intraventricular conduction resulting from the pacing-induced prolongation of the QRSd with a LBBB morphology.

\section{The impact of QRS change induced by biventricular pacing}

Previous reports investigating the association of paced QRSd and the outcome of CRT revealed similar results. Lecoq et al. evaluated CRT response in $139 \mathrm{CRT}$ patients and found that shortening of the QRSd by biventricular pacing was the single reliable predictor for a positive CRT response. ${ }^{5}$ The results of the PROSPECT trial also established that a positive outcome after CRT based on improved clinical composite scores and reductions in LV volumes were strongly related to the QRSd on the paced ECG, as well as the extent of QRS change induced by biventricular pacing. ${ }^{8}$ Similarly, a retrospective study by Rickard et al. revealed that QRS narrowing as indexed to baseline was significantly associated with reversed LV remodelling after CRT. ${ }^{9}$

Approximately one-third of patients do not derive clinical and/or echocardiographic benefit from biventricular pacing, which is also called 'non-response'. 10,11 Failure of CRT to induce reverse remodelling in these populations may be explained by persistent electrical dyssynchrony due to a prolonged QRSd which triggers asynchronous ventricular contractions, resulting in haemodynamic deterioration. Karaca et al. investigated the association of persistent electrical dyssynchrony (reflected by a prolonged QRSd on the paced ECG) with post-implantation echocardiographic mechanical dyssynchrony..$^{12}$ They found that prolonged QRS values correlated with higher Yu index values 
and that a biventricularly paced QRSd $>10$ ms compared to the baseline value was predictive of persistent mechanical dyssynchrony after CRT.

\section{Paced QRS duration and functional mitral regurgitation severity after cardiac resynchronization therapy}

Functional mitral regurgitation (FMR) is a common clinical entity associated with poor prognosis in heart failure. ${ }^{13,14}$ CRT is a proven therapy that reduces the severity of FMR by positively interacting with the underlying mechanisms, such as the increased tethering forces (due to papillary muscle dyssynchrony and/or annular dilatation) and the impaired closing forces (due to reduced LV contractility and/or LV dyssynchrony). ${ }^{15-17}$ Electrical resynchronization within the LV that subsequently results in reversal of LV/mitral remodelling and of LV/ papillary muscle dyssynchrony is the suggested mechanism of action that is associated with CRT-induced reduction in FMR. ${ }^{17}$ Significant FMR following CRT is associated with poor long-term prognosis as shown by Onishi et al. ${ }^{18}$ Accordingly, Porciani et al. reported that patients with FMR improvement after CRT derived more benefit in terms of LV reverse remodelling compared with those with no change or worsening of FMR. ${ }^{16}$ Although the association between the baseline electrical dyssynchrony (reflected by a prolonged QRSd) and the severity of FMR is well established in patients with dilated cardiomyopathy, ${ }^{19}$ the impact of CRT-induced change in QRSd on the severity of FMR has been less often addressed. A recent study by Karaca et al. showed that QRS narrowing after CRT was strongly correlated with reduction in FMR severity and was independently predictive of FMR improvement. ${ }^{20}$ Current knowledge supports the view that mitral valve remodelling induced by CRT is reflected by the magnitude of QRS change achieved by biventricular pacing that clearly is associated with improvements in LV mechanics, resulting in reduced FMR.

\section{Native QRS duration following cardiac resynchronization therapy}

Restoration of dyssynchronous electrical conduction within the HisPurkinje system by means of biventricular pacing has been proposed as the mechanism of action that correlates with the clinical and echocardiographic improvements after CRT. ${ }^{21,22}$ Reversal of electrical dyssynchrony with CRT is determined by the degree of narrowing of the native QRSd. Dizon et al. showed that CRT-induced loss of LBBB on the native ECG reflected improved electrical conduction that was subsequently associated with super-response to CRT suggesting that reversal of electrical remodelling is the main target of biventricular pacing in heart failure. ${ }^{23}$ Narrowing of the native QRS duration as a marker of reversed electrical remodelling (RER) after CRT has been shown to correlate with favourable structural changes and CRT response in previous studies. ${ }^{22,24}$ Yang et al. assessed native conduction during the follow-up of 74 CRT recipients by programming the device pacing-off mode to obtain the spontaneous ECG. ${ }^{22}$ They observed that patients with a narrowed intrinsic QRSd compared to baseline value seemed to derive more benefit in terms of CRT response and improvement in left ventricular ejection fraction. Similarly, Tereshchenko et al. reported that reversal of electrical remodelling was associated with improved survival and reduced arrhythmic events after CRT. ${ }^{25}$ Reversal of the intrinsic electrical dyssynchrony induced by CRT is the suggested mechanism of action that triggers the reversal of both mechanical dyssynchrony and LV remodeling, leading to favourable clinical and echocardiographic outcomes after CRT. However, there is no uniform definition of reversed electrical remodelling in the litareture. 9,22,26 Current knowledge suggests that absolute narrowing of the intrinsic QRSd is a surrogate marker to assess improvement in native conduction induced by biventricular pacing therapy. Translation of the native QRSd into clinical practice has been recently searched by a prospective study in 110 CRT recipients. ${ }^{37}$ The investigators showed that patients with "reversed electrical remodelling", in whom native QRSd was narrowed compared to the baseline value, were more likely to benefit from CRT in terms of major outcome variables such as echocardiographic response, FMR improvement, hospitalisation and death at mid-term follow-up. However, given the lack of long-term data regarding the impact of native QRSd on prognosis, future randomised studies are warranted to establish a follow-up algorithm including the assessment of the native ECG after CRT.

\section{Echocardiogram-based device optimisation}

CRT optimisation is a step-by-step process that should be initiated at the time of device implantation by proper lead localisation. The LV lead is recommended to be implanted at the latest activated segment to induce electrical synchronicity.27,28 Areas of scar tissue should be avoided in order to achieve efficient pacing. ${ }^{29-31}$ Apart from lead localisation, achievement of an almost 100\% biventricular pacing rate is a major concern to derive benefit from CRT devices. ${ }^{32,33}$ The clinical conditions that impair high pacing rates, such as atrial fibrillation and ventricular ectopic beats should be routinely searched and treated during the followup of CRT recipients. ${ }^{32,33}$ Appropriate device programming regarding the atrioventricular (AV) and interventricular (VV) intervals is essential to achieve optimal diastolic filling and stroke volume that is associated with favourable outcomes after CRT. ${ }^{34-36}$ All of the above-mentioned optimisation steps are known to affect the paced QRSd following device implantation. Future studies investigating the effect of each optimisation method on the QRSd would help establish a proper algorithm for device implantation, programming, and follow-up. Currently, only patients with clinical evidence of CRT non-response are recommended to undergo device optimisation, rather than performing device programming (especially the AV and $\mathrm{V}$ intervals) routinely after implantation. ${ }^{3}$ We strongly suggest that early post-operative evaluation of pacing intervals is mandatory and should be a part of routine follow-up assessment. Given the proven benefit of targeting the narrowest possible QRSd on clinical outcomes after CRT, comprehensive baseline device optimisation combined with ECG-based assessment of the AV and $\mathrm{V}$ delays is a reliable strategy to improve 'responsiveness' following CRT.

\section{Conclusion}

The failing heart, which exhibits delayed intra-ventricular conduction time reflected by a prolonged QRSd can be 'resynchronized' by biventricular pacing. Minimising the time delay for the onset of activation within the left ventricular segments by CRT results in synchronised contractions that further induce reversed remodelling in both the ventricle, the mitral valve, and the conduction system. Prognosis after CRT is closely related to the extent of resynchronization that can be simply reflected by the extent of QRS narrowing on the ECG. Moreover, the native QRSd is a surrogate marker of reversed electrical remodelling induced by CRT that has prognostic implications.

In conclusion, the ECG (especially the QRSd) is the single most important clinical tool in the era of resynchronization for, not only selecting the CRT candidates, but also for device optimisation, assessment of efficient pacing, estimation of response, and determining prognosis. Future CRT guidelines should provide recommendations on the routine assessment of 'paced' and the 'native' QRS durations in the follow-up algorithms of CRT recipients. 
1. Cleland JG, Freemantle N, Erdmann E, et al., Long-term mortality with cardiac resynchronization therapy in the Cardiac Resynchronization-Heart Failure (CARE-HF) trial, Eur J Heart Fail, 2012;14:628-34

2. Goldenberg I, Hall WJ, Beck CA, et al., Reduction of the risk of recurring heart failure events with cardiac resynchronization therapy: MADIT-CRT (Multicenter Automatic Defibrillator Implantation Trial With Cardiac Resynchronization Therapy), J Am Coll Cardiol, 2011;58:729-37.

3. Brignole M, Auricchio A, Baron-Esquivias G, et al., 2013 ESC guidelines on cardiac pacing and cardiac resynchronization therapy: the task force on cardiac pacing and resynchronization therapy of the European Society of Cardiology (ESC), Europace, 2013;15:1070-118.

4. Foley PW, Leyva F, Frenneaux MP, What is treatment success in cardiac resynchronization therapy?, Europace, 2009;11:58-65

5. Lecoq G, Leclercq C, Leray E, et al., Clinical and electrocardiographic predictors of a positive response to cardiac resynchronization therapy in advanced heart failure, Eur Heart J, 2005;26:1094-100.

6. Miyoshi F, Kobayashi Y, Itou H, et al., Prolonged paced QRS duration as a predictor for congestive heart failure in patients with right ventricular apical pacing, Pacing Clin Electrophysiol, 2005;28:1182-8.

7. Chen $S$, Yin $Y$, Lan $X$, et al., PREDICT-Heart Failure study international group. Paced QRS duration as a predictor for clinical heart failure events during right ventricular apical pacing in patients with idiopathic complete atrioventricular pacing in patients with idiopathic complete atrioventricular block: results from an observationa,

8. Hsing JM, Selzman KA, Leclercq C, et al., Paced left ventricular QRS width and ECG parameters predict outcomes after cardiac resynchronization therapy: PROSPECT-ECG substudy, Circ Arrhythm Electrophysiol, 2011;4:851-7.

9. Rickard J, Popovic Z, Verhaert D, et al., The QRS narrowing index predicts reverse left ventricular remodeling following cardiac resynchronization therapy, Pacing Clin Electrophysiol, 2011;34:604-11.

10. Chatterjee NA, Singh JP, Cardiac resynchronization therapy: past, present, and future, Heart Fail Clin, 2015;11:287-303.

11. Steffel J, Ruschitzka F Superresponse to cardiac resynchronization therapy, Circulation, 2014;130:87-90.

12. Karaca O, Omaygenc $\mathrm{O}$, Cakal B, QRS prolongation after cardiac resynchronization therapy is a predictor of persistent mechanical dyssynchrony, I Interv Card Electrophysiol, 2015: [Epub ahead of print]

13. Pino PG1, Galati A, Terranova A, Functional mitra regurgitation in heart failure, I Cardiovasc Med (Hagerstown),
2006; 7:514-23

14. Asgar AW, Mack MJ, Stone GW, Secondary mitral regurgitation in heart failure: pathophysiology, prognosis, and therapeutic considerations, J Am Coll Cardiol, 2015;65:1231-48.

15. Vinereanu D, Mitral regurgitation and cardiac resynchronization therapy, Echocardiography, 2008;25:1155-66.

16. Porciani MC, Macioce R, Demarchi $G$, et al.. Effects of cardiac resynchronization therapy on the mechanisms underlying functional mitral regurgitation in congestive heart failure Eur J Echocardiogr, 2006;7:31-9.

17. Ypenburg $C$, Lancellotti $P$ Tops $L F$, et al., Mechanism of improvement in mitral regurgitation after cardiac resynchronization therapy, Eur Heart J, 2008:29:757-65.

18. Onishi T, Onishi T, Marek JJ, et al., Mechanistic features associated with improvement in mitral regurgitation after cardiac resynchronization therapy and their relation to longterm patient outcome, Circ Heart Fail, 2013;6:685-93.

19. Barbieri A, Grigioni F, Bursi F, Role of severe functional mitral regurgitation in predicting electrical remodeling in idiopathic dilated cardiomyopathy, I Cardiovasc Med (Hagerstown), 2006;7:691-5.

20. Karaca O, Omaygenc MO, Cakal B, et al., Effect of QRS Narrowing After Cardiac Resynchronization Therapy on Functional Mitral Regurgitation in Patients With Systolic Heart Failure, Am J Cardiol, 2016;117:412-9.

21. Sassone B, Gambetti S, Bertini M, et al., Relation of QRS duration to response to cardiac resynchronization therapy, Am I Cardiol, 2015:115:214-9.

22. Yang $X W$, Hua $W$, Wang J, et al. Native QRS narrowing reflects electrical reversal and associates with anatomica reversal in cardiac resynchronization therapy, I Interv Card Electrophysiol, 2014;41:161-8.

23. Dizon J, Horn E, Neglia J, et al., Loss of left bundle branch block following biventricular pacing therapy for heart failure: evidence for electrical remodeling?, I Interv Card Electrophysiol, 2004;10:47-50

24. Boriani G, Biffi M, Martignani C, et al., Electrocardiographic remodeling during cardiac resynchronization therapy, Int Cardiol, 2006;108:165-70

25. Tereshchenko LG, Henrikson CA, Stempniewicz P, et al., Antiarrhythmic effect of reverse electrical remodeling associated with cardiac resynchronization therapy, Pacing Clin Electrophysiol, 2011;34:357-64.

26. Yang XW, Hua W, Wang J, et al., Regression of fragmented QRS complex: a marker of electrical reverse remodeling in cardiac complex: a marker of electrical reverse remodeling in cardlac 2015;20:18-27.

27. Dong $Y X$, Powell $B D$, Asirvatham $\mathrm{SJ}$, et al., Left ventricular lead position for cardiac resynchronization: a comprehensive cinegraphic, echocardiographic, clinical, and survival analysis, Europace, 2012;14:1139-47.

28. Chen L, Tiongson JE, Obrzut S, et al., Standard ches radiograph predicts left ventricular lead location in chronic resynchronization therapy patients more accurately than intraoperative fluoroscopy, J Interv Card Electrophysiol, 2012;35:323-30.

29. Huntjens PR, Walmsley J, Ploux S, et al., Influence of left ventricular lead position relative to scar location on response to cardiac resynchronization therapy: a model study, to cardiac resynchronization therapy:

30. de Roest GJ, Wu L, de Cock CC, et al., Scar tissue-guided left ventricular lead placement for cardiac resynchronization therapy in patients with ischemic cardiomyopathy: an acute pressure-volume loop study, Am Heart J, 2014;167:537-45.

31. Taylor RJ, Umar F, Panting JR, et al., Left ventricular lead position, mechanical activation, and myocardial scar in relation to left ventricular reverse remodeling and clinical outcomes after cardiac resynchronization therapy: A featuretracking and contrast-enhanced cardiovascular magnetic resonance study, Heart Rhythm, 2016;13:481-9.

32. Ruwald MH, Mittal S, Ruwald AC, et al, Association between frequency of atrial and ventricular ectopic beats and biventricular pacing percentage and outcomes in patients with cardiac resynchronization therapy, J Am Coll Cardiol, 2014;64:971-81.

33. Ousdigian KT, Borek PP, Koehler JL, et al., The epidemic of inadequate biventricular pacing in patients with persistent or permanent atrial fibrillation and its association with mortality, Circ Arthym Electrophysiol, 2014:7:370-6.

34. van Gelder BM, Bracke FA, Meijer A, et al., Effect of optimizing the $\mathrm{V} V$ interval on left ventricular contractility in cardiac resynchronization therapy, Am J Cardiol, 2004;93:1500-3.

35. Arbelo E, Tolosana JM, Trucco E, et al., Fusion-optimized intervals (FOI): a new method to achieve the narrowest QRS for optimization of the AV and VV intervals in patients undergoing cardiac resynchronization therapy, J Cardiovasc Electrophysiol, 2014;25:283-92.

36. van Deursen CJ, Blaauw Y, Witjens Ml, et al., The value of the 12-lead ECG for evaluation and optimization of cardiac resynchronization therapy in daily clinical practice, $J$ Electrocardiol, 2014:47:202-11.

37. Karaca O, Cakal B, Omaygenc MO, et al., Native Electrocardiographic QRS Duration after Cardiac Resynchronization Therapy: the Impact on Clinical Outcomes and Prognosis, I Card Fall, 2016;Article in press, do: 10.1016/j.cardfail.2016.04.001 